\title{
Surgical treatment of a ventricular aneurysm in a patient with essential thrombocythemia complicated by acute myocardial infarction: A case report
}

\author{
DONG-WEN WANG, YONG ZHANG, JIAN-MIN YAO and ZHI-BIN XIAO \\ Department of Cardiovascular Surgery, Beijing Military Region General Hospital, Beijing 100700, P.R. China
}

Received July 6, 2013; Accepted October 29, 2013

DOI: $10.3892 /$ etm.2013.1389

\begin{abstract}
Essential thrombocythemia (ET) is a chronic clonal myeloproliferative disorder, which is often complicated by arterial or venous thrombosis and idiopathic bleeding diathesis. The present study reports a female patient with ET complicated by acute myocardial infarction, leading to ventricular aneurysm following interventional therapy for 3 years and a subsequent in-stent restenosis. Following careful examination, a ventricular aneurysm resection and coronary artery bypass graft were carried out. During this case, the monitoring and controlling of the platelet count, preand post-operatively, was extremely important for successful surgery.
\end{abstract}

\section{Introduction}

Essential thrombocythemia (ET) is a chronic clonal myeloproliferative disorder, which is characterized by persistent thrombocytosis and complicated by arterial or venous thrombosis and idiopathic bleeding diathesis. These complications are the primary causes of mortality in patients with ET (1-3). Although ET-related thrombosis is uncommon in coronary arterial thrombosis, it is more common in extremity vascular thrombosis and may subsequently cause acute myocardial infarction (AMI). Cases of ET complicated with AMI have been reported previously, but have not been treated with surgical therapy (4-6). The present case study reports a patient with ET complicated by AMI, leading to ventricular aneurysm following interventional therapy for 3 years and subsequent in-stent restenosis. Following careful examination, a ventricular aneurysm resection and coronary artery bypass graft were carried out.

Correspondence to: Professor Dong-Wen Wang, Department of Cardiovascular Surgery, Beijing Military Region General Hospital, Beijing 100700, P.R. China

E-mail: wangdongwendr@yeah.net

Key words: essential thrombocythemia, acute myocardial infarction, ventricular aneurysm, surgical treatment

\section{Case report}

The present patient was a 48-year-old female who had been admitted to Beijing Tiantan Hospital (Beijing, China) 2 years previously and had complained of a persistently congested chest and radiated pain in her left shoulder for a period of 6 hours. Electrocardiography revealed AMI of the anterior wall. The biomarkers of myocardial injury were significantly increased (troponin I, $12.27 \mathrm{ng} / \mathrm{ml}$; creatine kinase, 1,548 IU/1; and creatine kinase isozyme CK-MB, $189.0 \mathrm{U} / \mathrm{l}$ ) compared with the normal range (troponin I, <0.04 ng/ml; creatine kinase, 24-170 IU/1; and CK-MB, 0.97-2.88 ng/ml). Laboratory tests revealed that levels of leukocytes $\left(7.1 \times 10^{9} / 1\right)$, hemoglobin (128 g/l), high density lipoprotein (1.20 mmol/l), low density lipoprotein $(2.51 \mathrm{mmol} / 1)$ and fasting blood-glucose $(5.4 \mathrm{mmol} / \mathrm{l})$ were in the normal range. However, platelet levels $\left(8.89 \times 10^{11} / 1\right)$ were higher than the normal range $\left(1-3 \times 10^{11} / 1\right)$. The patient was treated using primary percutaneous coronary intervention. Intraoperative observations indicated that $90 \%$ of the near front descending branch was narrowed and the remainder of the aortic wall was smoothed. Subsequently, a rapamycin-eluting stent $(3.5 \times 29 \mathrm{~mm})$ was embedded in the front descending branch (Fig. 1A, B and C). Postoperatively, chest congestion was relieved and the patient was discharged. The patient was initially administered with clopidogrel ( $75 \mathrm{mg}$ per day) to reduce the platelet count, which was subsequently replaced with enteric aspirin 1 year later (100 mg per day).

The patient complained of palpitations and shortness of breath one month prior to the second admission. During this period, the patient experienced paroxysmal nocturnal dyspnea and was unable to lie in a horizontal position at night. High blood pressure, diabetes or hyperlipoidemia were not observed or a history of smoking. The patient had been diagnosed with ET in the Beijing Tiantan Hospital 15 years previously. All routine test references were within the normal range, with the exception of the platelet count $\left(5.52 \times 10^{11} / 1\right)$. Color doppler echocardiography was performed and identified a ventricular aneurysm. Furthermore, coronary arteriography revealed a 50\% narrowing in the front descending stent and the formation of a left ventricular aneurysm (Fig. 1D and E). The symptoms of cardiac insufficiency disappeared following symptomatic treatment. The patient was administered hydroxyurea (100 mg per day) for 
A

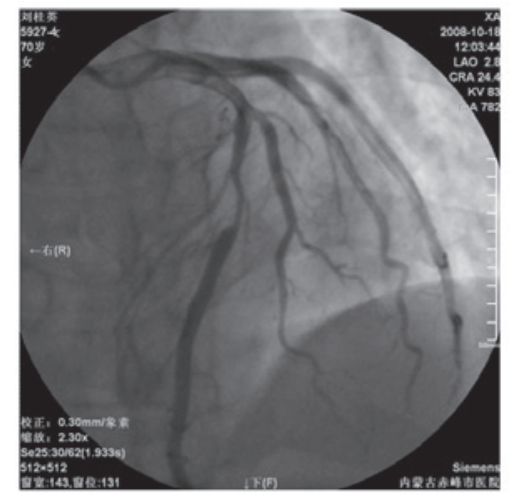

D

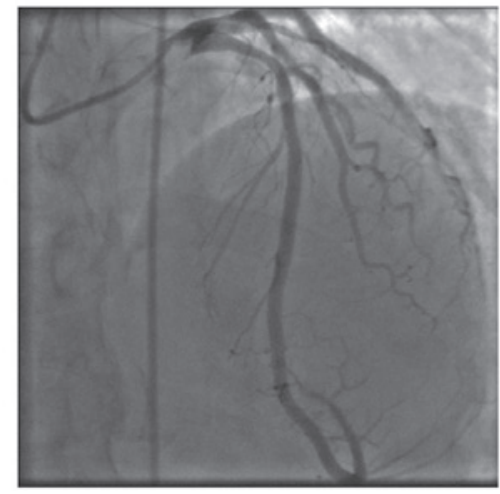

B

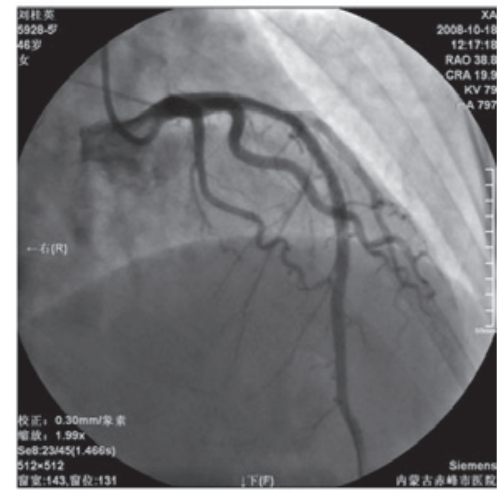

$\mathbf{E}$

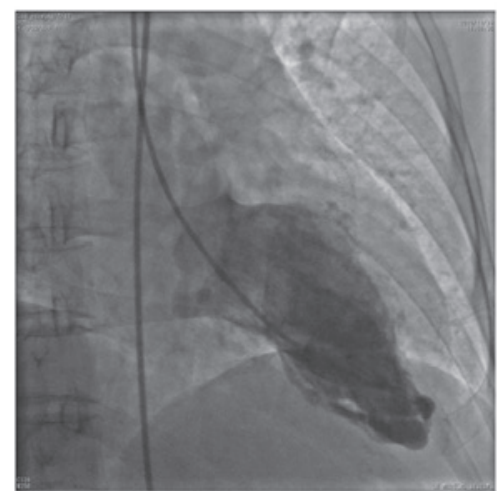

C

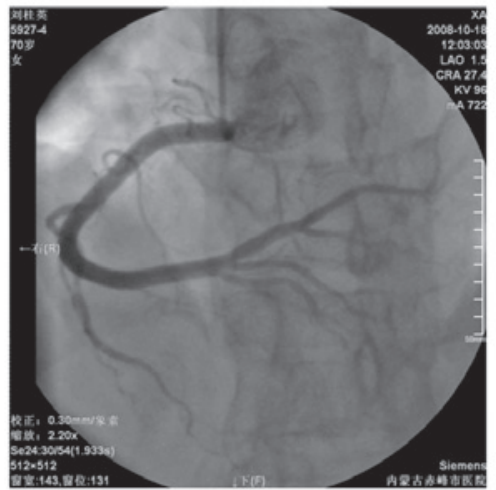

F

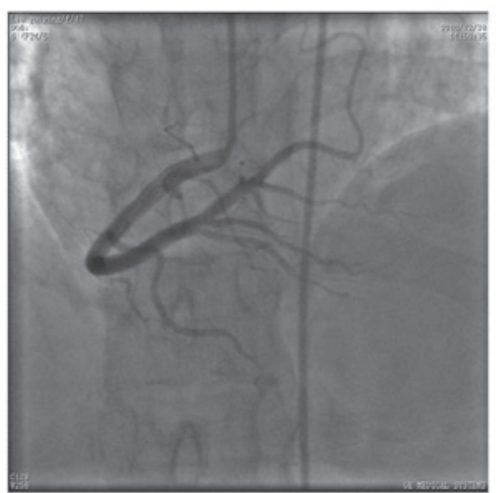

Figure 1. Coronary angiography of an essential thrombocythemia case with acute myocardial infarction. Examination results of the (A-C) first and (D-F) second time admissions.

a week in order to reduce platelet count to $325 \times 10^{9} / 1$. The ventricular aneurysm was resected under general anesthesia and cardiopulmonary bypass. Bypass surgery was performed between the left internal mammary artery and the front descending stent. The patient recovered well and no complications, for example hemorrhage or thrombosis, occurred. The platelet count was controlled using hydroxyurea (100 mg per day) and aspirin (100 mg per day). At $~ 15$ days following surgery, the platelet count was $286 \times 10^{9} / 1$ and the patient was discharged. At the 3-month follow-up, the patient showed no signs of heart insufficiency or angina. An ultrasonic cardiogram showed that the left ventricular aneurysm had disappeared. Furthermore, no heart-related symptoms were detected at a 2-year follow-up. The study was approved by The Ethics Committee of Being Military Region General Hospital, Beijing, China. Written informed patient consent was obtained from the patient or the patient's family.

\section{Discussion}

The annual incidence of ET is $\sim 1.5$ per 100,000 individuals worldwide (7). The principal causes of morbidity and mortality in ET are thrombosis, hemorrhage and progression to myelofibrosis or acute myelogenous leukemia (8). Only $9.4 \%$ of patients with ET have myocardial infarction (9). Although the median age of patients with ET is 60 years, $10-25 \%$ of patients are $<40$ years of age and one-third of patients are asymptomatic (10-13). With the exception of a history of thrombosis, cardiovascular disease risk factors, including smoking, high blood pressure, hyperlipidemia and diabetes, are also risk factors for thrombosis (12).
In the present report, we describe an ET case complicated with AMI and the formation of a ventricular aneurysm. Preoperative examinations showed no cardiovascular disease risk factors and the vital organs were healthy. Therefore, a history of ET was the only surgical risk. Although the incidence rate of thrombosis is higher than bleeding for ET cases, the risk of bleeding is relatively higher in surgical cases (14). Regardless of the complications of thrombosis or hemorrhage, the monitoring and control of the platelet count, preoperatively or postoperatively, is key for a successful surgery. In this case, the patient maintained a relatively normal platelet count as a result of treatment with hydroxyurea and the platelet count in the perioperative period was consistent with previous case reports (15-17). Meanwhile, such alteration of the platelet count may also be controlled postoperatively.

Similar cases have been reported in previous studies. However, the majority of such cases were treated with an interventional approach and rarely via surgical methods. ET, complicated by coronary artery thrombosis, has no uniform treatment guidelines worldwide and therefore clinical treatment is difficult. As well as utilizing anti-platelet drugs, including aspirin and clopidogrel, it is important to use platelet-cytoreductive therapy for ET patients (18-20). In the case of coronary artery stent restenosis, we recommend a combined treatment of hydroxyurea and aspirin to prevent thrombosis.

In conclusion, surgical treatment for ET with myocardial infarction is rare and no uniform treatment guidelines are currently available worldwide. Therefore, ET is a key risk in surgery. It is important to prevent thrombosis and hemor- 
rhage, as well as monitor and control the platelet count preoperatively and postoperatively in order to achieve a successful surgery.

\section{References}

1. Besses C, Cervantes F, Pereira A, Florensa L, Solé F, Hernández-Boluda JC, Woessner S, Sans-Sabrafen J, Rozman C and Montserrat E: Major vascular complications in essential thrombocythemia: a study of the predictive factors in a series of 148 patients. Leukemia 13: 150-154, 1999.

2. Weng S, Zhu X, Jin Y, Wang T and Huang H: Protective effect of erythropoietin on myocardial infarction in rats by inhibition of caspase-12 expression. Exp Ther Med 2: 833-836, 2011.

3. Schafer AI: Thrombocytosis. N Engl J Med 350: 1211-1219, 2004.

4. Mizuta E, Takeda S, Sasaki N, Miake J, Hamada T, Shimoyama M, Tajima F, Igawa $\mathrm{O}$, Shigemasa $\mathrm{C}$ and Hisatome I: Acute myocardial infarction in a patient with essential thrombocythemia: successful treatment with percutaneous transluminal coronary recanalization. Circ J 69: 1000-1002, 2005.

5. Pick RA, Glover MU, Nanfro JJ, Dubbs WF, Gibbons JA and Vieweg WV: Acute myocardial infarction with essential thrombocythemia in a young man. Am Heart J 106: 406-407, 1983.

6. Zhang Z, Wan X, Liu Y, Lin X, Ni Z, Yang X and Zhang L: Non-ST-segment elevation myocardial infarction in a patient with essential thrombocythemia treated with glycoprotein IIb/IIIa inhibitor: a case report. Clin Appl Thromb Hemost 17: 532-534, 2011.

7. Johansson P: Epidemiology of the myeloproliferative disorders polycythemia vera and essential thrombocythemia. Semin Thromb Hemost 32: 171-173, 2006.

8. McIntyre KJ, Hoagland HC, Silverstein MN and Petitt RM: Essential thrombocythemia in young adults. Mayo Clin Proc 66: 149-154, 1991

9. Rossi C, Randi ML, Zerbinati P, Rinaldi V and Girolami A: Acute coronary disease in essential thrombocythemia and polycythemia vera. J Intern Med 244: 49-53, 1998.

10. Alvarez-Larrán A, Cervantes F, Bellosillo B, Giralt M, Juliá A, Hernández-Boluda JC, Bosch A, Hernández-Nieto L, Clapés V, Burgaleta C, Salvador C, Arellano-Rodrigo E, Colomer D and Besses C: Essential thrombocythemia in young individuals: frequency and risk factors for vascular events and evolution to myelofibrosis in 126 patients. Leukemia 21: 1218-1223, 2007.
11. Carobbio A, Finazzi G, Guerini V, Spinelli O, Delaini F, Marchioli R, Borrelli G, Rambaldi A and Barbui T: Leukocytosis is a risk factor for thrombosis in essential thrombocythemia: interaction with treatment, standard risk factors, and Jak2 mutation status. Blood 109: 2310-2313, 2007.

12. Wang Y, Zhang ZZ, Wu Y, Zhan J, He XH and Wang YL: Honokiol protects rat hearts against myocardial ischemia reperfusion injury by reducing oxidative stress and inflammation. Exp Ther Med 5: 315-319, 2013.

13. Wolanskyj AP, Schwager SM, McClure RF, Larson DR and Tefferi A: Essential thrombocythemia beyond the first decade: life expectancy, long-term complication rates, and prognostic factors. Mayo Clin Proc 81: 159-166, 2006.

14. Ruggeri M, RodeghieroF, Tosetto A, Castaman G,ScognamiglioF, Finazzi G, Delaini F, Micò C, Vannucchi AM, Antonioli E, De Stefano V, Za T, Gugliotta L, Tieghi A, Mazzucconi MG, Santoro C and Barbui T; Gruppo Italiano Malattie Ematologiche dell'Adulto (GIMEMA) CHronis Myeloproliferative Diseases Working Party: Postsurgery outcomes in patients with polycythemia vera and essential thrombocythemia: a retrospective survey. Blood 111: 666-671, 2008.

15. Das SS, Bose S, Chatterjee S, Parida AK and Pradhan SK: Thrombocytapheresis: managing essential thrombocythemia in a surgical patient. Ann Thorac Surg 92: e5-e6, 2011.

16. Jin D, Wu Y, Zhao L, Guo J, Zhang K and Chen Z: Atorvastatin reduces serum HMGB1 levels in patients with hyperlipidemia. Exp Ther Med 4: 1124-1126, 2012.

17. Ohto T, Shihara H, Miyauchi Y and Nakajima N: A case of coronary artery bypass surgery using left internal thoracic artery and right gastroepiploic artery for a patient with essential thrombocythemia. Jpn J Thorac Cardiovasc Surg 46: 767-771, 1998 (In Japanese).

18. Lv H and Ning B: Pathogenesis of bloodstream infection in children with blood cancer. Exp Ther Med 5: 201-204, 2013.

19. Harrison CN, Campbell PJ, Buck G, Wheatley K, East CL, Bareford D, Wilkins BS, van der Walt JD, Reilly JT, Grigg AP, Revell P, Woodcock BE and Green AR; United Kingdon Medical Research Council Primary Thrombocythemia 1 Study: Hydroxyurea compared with anagrelide in high-risk essential thrombocythemia. N Engl J Med 353: 33-45, 2005.

20. Vannucchi AM, Guglielmelli P and Tefferi A: Advances in understanding and management of myeloproliferative neoplasms. CA Cancer J Clin 59: 171-191, 2009. 